\title{
Item to decision mapping in rapid response learning
}

\author{
DAVID M. SCHNYER \\ Boston VA Healthcare System, Boston, Massachusetts \\ Boston University School of Medicine, Boston, Massachusetts \\ and Athinoula A. Martinos Center for Biomedical Imaging, Charlestown, Massachusetts
}

IAN G. DoBbins

Duke University, Durham, North Carolina

Lindsay Nicholls, Sarah Davis, ANd MieKe Verfaellie
Boston VA Healthcare System, Boston, Massachusetts

AND

DANIEL L. SCHACTER

Harvard University, Cambridge, Massachusetts

\begin{abstract}
Repeated classification of a visually presented stimulus rapidly leads to a form of response learning that bypasses the original evaluation in favor of a more efficient response mechanism. In two experiments, we examined the level of input and output representations that make up this form of learning. In Experiment 1, alterations in the finger mapping of the output response had no effect on the expression of response learning, demonstrating that a classification decision, not motor output, is associated with repeated items. In Experiments $2 \mathrm{~A}$ and $2 \mathrm{~B}$, we tested whether response learning would transfer across different visual exemplars of a studied item. There was no evidence of transfer to different visual exemplars, even when these exemplars were judged to be highly visually similar. Taken together, these results indicate that response learning consists of the formation of an association between a specific visual representation and a classification decision.
\end{abstract}

A previous encounter with an item will often result in changes in a person's ability to identify, produce, or classify that item, referred to as repetition priming. Simple changes in presentation format or task demands between exposures can have significant effects on the magnitude of priming (Schacter, Dobbins, \& Schnyer, 2004). The nature and type of these specificity effects may be critical in helping to identify the representational level, or levels, upon which behavioral facilitation rests. For example, if a repetition priming effect were severely disrupted by changing the nature of the manual response or by changing from a manual to a verbal response, this finding would suggest that the learning or facilitation occurred at a relatively late stage of processing. Despite the known sensitivity of priming to a variety of test manipulations (Burgund \& Marsolek, 1997; Vaidya, Gabrieli, Verfaellie, Fleischman, \& Askari, 1998), there have been relatively few attempts to delineate the mechanisms that are responsible for these effects. In a recent series of studies (Dobbins, Schnyer, Verfaellie, \& Schacter, 2004; Schnyer, Dobbins, Nicholls, Schacter, \& Verfaellie, 2006), we have begun to explore the cognitive and neural mechanisms resulting in one type of priming specificity that suggests that the rapid learning of decision outcomes or responses may be a significant part of typically observed priming gains. We have referred to this phenomenon as response learning.

\section{Previous Examinations of Response Learning}

In previous studies, we have examined response learning by utilizing a semantic classification task in which the framing of the decision cue was changed between initial exposure (study) and subsequent primed presentations (test). During a study period, subjects were asked to indicate whether visually presented common objects were "bigger than a shoebox." Items were presented during study either once or three times. At test, the subjects continued making size decisions to objects repeated from study and to new objects. The test phase was conducted either with the same decision cue as that presented during study ("bigger than a shoebox") or with an inversion of the decision cue ("smaller than a shoebox"). The effect of repetition on items previously presented once or three times was compared between the two decision cue conditions. We postulated that if the facilitation associated with

D. M. Schnyer, schnyer@psy.utexas.edu 
repetition reflected small modifications in object identification and/or knowledge representations within the same object-processing stream as that engaged when the items were first presented, inversion of the decision cue would cause little disruption in priming. That is, if priming were the result of learning or tuning in these representations (Wiggs \& Martin, 1998), its expression should not be particularly sensitive to the change in decision cue. However, if the results demonstrated that priming was significantly disrupted or eliminated by a change in the decision cue, this would suggest that priming reflected the fact that the subjects rapidly associated their prior responses or decisions with each item and, thereby, bypassed many of the more deliberative processes engaged when an item was first presented.

The results provided evidence for the latter view (Dobbins et al., 2004). Cue inversion disrupted a significant portion of the response facilitation associated with repetition. Moreover, as indicated by fMRI, cue inversion completely eliminated the neural-priming signature across several cortical areas where repetition-related reductions in neural activity were evident, including the fusiform gyrus and left prefrontal regions. In addition, regression analysis demonstrated that changes in the prefrontal cortex predicted the level of behavioral facilitation during learning and the magnitude of behavioral disruption that would later occur when the cue was inverted. Given this outcome, we tentatively suggested that the behavioral facilitation and neural activity reductions, both in regions associated with early visual processing and in those associated with later classification and decision-making processes, resulted from the learning of prior responses or decisions that enabled the observers to bypass controlled or deliberative classification processes that would be engaged when an item was first encountered. This view differs significantly from one offered in previous fMRI studies of repetition priming (Buckner et al., 1998; Koutstaal et al., 2001), postulating that the facilitation resulting from multiple repetitions reflects continued tuning of object identification and knowledge systems.

In a subsequent set of behavioral experiments, Schnyer et al. (2006) replicated the finding that the level of priming was reduced with cue inversion from that seen when the cue orientation was maintained from study to test. Unlike the fMRI study, in which cue sensitivity was apparent primarily for items repeated three times, here cue inversion disrupted priming for items repeated once, as well as for those repeated three times. Moreover, in this experiment, it was apparent that all the added facilitation associated with multiple repetitions was eliminated by cue inversion. In a second experiment, the effect of cue inversion was tested in amnesic patients with damage to the medial temporal lobe (MTL). These patients revealed a significant behavioral-priming effect but no evidence of a response-learning component. Unlike controls, the MTL amnesics did not reveal added facilitation with multiple repetitions, and the observed priming was not disrupted by cue inversion. This study led to the conclusion that rapid response or decision learning reflects an MTL-dependent associative-learning mechanism whereby a particular re- sponse or decision outcome becomes associated with an item.

It is important to note, however, that the presence of cue specificity effects, as documented above, does not imply that all of the behavioral facilitation observed in classification priming is dependent upon the same mechanism. Indeed, although a considerable portion of the behavioral facilitation was dependent upon the format of the decision cue, there was nonetheless a portion of repetition-induced facilitation that appeared independently of the specific format of the cue and that was not impaired in amnesia. These findings suggest that priming, even within a single task, may reflect multiple mechanisms or processes, only one of which reflects the learning of prior decisions or responses. In this way, our results and conclusions differ from previous demonstrations of response learning that have emphasized a common underlying mechanism of instance learning that is solely responsible for repetition priming (Logan, 1990; Logan \& Etherton, 1994; Logan, Taylor, \& Etherton, 1996).

\section{Delineating the Input/Output Levels Bound in Response Learning: The Present Experiments}

The focus of the present experiments was to pin down the input and output levels of the bound association that is formed in rapid response learning. On the output side, the question arises as to whether the effect reflects the learning of an association between an item and a specific motor/finger response or, rather, between an item and a specific classification/decision. So far, the evidence from previous studies has been mixed. In many of the previous studies of instance learning, motor mapping has been assumed to play a minimal role (Dennis \& Schmidt, 2003; Fisk \& Schneider, 1984; Logan, 1990). Instead, it has been assumed that instance learning reflects the formation of an association between items and response categories or classification actions (Logan, 1990). More recently, however, it has been demonstrated that conditions can be implemented that result in interactions between motor mapping and other features of the learned association (Logan et al., 1996).

The role of motor mapping in the object classification task that we have utilized in our previous studies is also unclear. In Dobbins et al. (2004), subjects were tested with a version of the task outside of the MRI scanner that left the decision cue intact ("bigger than a shoebox") but reversed the assignment of yes and no response buttons across study and test. In that experiment, a sizable priming disruption occurred, but more important, there was no statistical interaction between the effect of decision cue inversion and the effect of motor mapping inversion. This result suggested that the form of response learning exhibited in the object classification task is predominantly driven by motor associations and conflicted with some of the previous studies mentioned above.

There are a number of reasons to be skeptical of our previous motor-mapping results. First, the experimental design utilized in that study (which was adapted so as to parallel the one used during the fMRI experiment) contained several suboptimal methodological features. The critical 
comparison between the same-cue and the inverted-cue conditions suffered from a temporal confound, in that the inverted-cue condition was tested later in the experiment than was the same-cue condition. In addition, items in the inverted-cue condition had received one extra repetition, in comparison with those in the original cue condition. Any difference between study and test blocks could be driven, in part, by changes in response speed with time or the additional repetition. Given this ambiguity, it is not possible to draw any firm conclusions about whether the output level in the classification task is bound to the motor output or the decision. Experiment 1 was designed to address this issue.

The goal of Experiment 2 was to determine the input level of response learning by examining the perceptual specificity of the effect, given evidence that priming is often perceptually highly specific (Koutstaal et al., 2001). If the response-learning phenomenon appears to be tied to highly specific perceptual representations, it is possible that the disruptions in priming across perceptual manipulations in earlier research (Schacter et al., 2004) might, in some cases, have been due to disruptions of response learning. Although a number of studies have examined object priming across different visual exemplars (Biederman \& Gerhardstein, 1993; Koutstaal et al., 2001; Roediger \& Srinivas, 1993), there are no studies that have tested whether the priming preserved across object exemplars is or is not sensitive to the format of the decision cue. Thus, the level of perceptual specificity of the response-learning mechanism is unknown.

\section{EXPERIMENT 1}

In Experiment 1, we evaluated the effect of changes in motor mapping on priming in the classification paradigm used in our previous work (Schnyer et al., 2006) by introducing a switch in the finger mapping of the yes and no response keys. If a simple change in finger assignment resulted in a significant reduction in priming, as does cue inversion, this finding would support the view that during response learning, items become associated with specific motor operations. Alternatively, if a switch in finger mapping had no significant effect on response learning, it would suggest that during response learning, items become associated with a specific classification (e.g., bigger than).

\section{Method}

In order to compare directly the results of this experiment with those in our previous examination of response learning, the stimuli, paradigm, and procedure were exactly the same as those in Schnyer et al. (2006). The only difference was that rather than inversion of the decision cue, the subjects were asked to invert which finger they used to indicate the yes and no responses.

Subjects. Sixteen young native speakers of English ( 2 of them male, 14 female), with normal or corrected-to-normal vision, took part in the experiment. The subjects (mean age $=21$ years; range, 18-25) were recruited through flyers and advertisements at local colleges and universities and received $\$ 10$ for their participation. The subjects were screened using a short medical questionnaire to ensure that they were free from current psychiatric or neurologi- cal disorder, any history of brain injury, or excessive drug or alcohol use. Written informed consent was obtained from each subject prior to the experimental session. The Human Subjects Committees of Boston University School of Medicine and the Veterans Affairs Healthcare System approved all the procedures.

Stimuli. Four hundred eight colored line drawings of common animate and inanimate objects were selected from commercially available clip art collections (CD-ROM from Corel Mega Gallery, Corel Corporation, 1997). The pictures reflected varying orientations and visual size. The stimuli were presented on a Mac Powerbook laptop computer using Psyscope 1.2.5 (Carnegie Mellon University, 1994). The objects were presented within a centrally located $8.75 \times 8.75 \mathrm{~cm}$ box, and viewing was approximately $75 \mathrm{~cm}$ from the screen, resulting in a vertical and horizontal visual angle subtending approximately $6^{\circ}-7^{\circ}$.

Procedure. A brief task instruction period was followed by four alternating study-test cycles. During the study phase of each cycle, 34 pictures were presented once, and 34 pictures were presented three times, for a total of 136 presentations. The items presented only once were evenly distributed throughout the study phase in such a way that one third of these items were encountered with each full repetition cycle of the items presented a total of three times. The subjects were asked to make a size judgment by deciding whether, in real life, the depicted object was "bigger than a shoe box." They indicated their decision by pushing a yes or a no key with the index or the middle finger, respectively, of their right hand. Following the study phase and a short pause, the subjects took part in a test phase consisting of two test blocks. Each test block consisted of 17 pictures presented once during study (low prime), 17 pictures presented three times during study (high prime), and 17 novel pictures. None of these pictures were repeated within or between test blocks. The test blocks differed only with regard to the finger mapping of the yes and no decisions made by the subjects: In one block, the subjects indicated their response with the same mapping ("<yes/no $>$ ") as that made during the study phase, whereas in the other block, the mapping was inverted ("< $<$ no/yes $>$ ") with respect to the finger that indicated each response. For two study-test cycles, the subjects used the same mapping in the first test block as that used in the study phase and the inverted mapping in the second test block, whereas for the other two cycles the order was switched.

The pictures were randomly assigned to one of the four study-test cycles. Within each cycle, the pictures in the test phase were rotated among the three possible conditions (novel, low prime, and high prime). In addition, the pictures in the test phase were rotated so that the pictures occurring in the same-cue condition for 1 subject occurred in the inverted-cue condition for another. This resulted in a total of six versions of the experiment.

The pictures were presented at a rate of one every $2 \mathrm{sec}$ and were accompanied at the bottom of the screen by the finger mapping to be used to indicate the size decision on that trial. Instructions appearing with the stimuli were "Bigger than a shoebox, $<$ yes/no $>$ " during the study phase and same mapping at test and "Bigger than a shoebox, $<$ no/yes $>$ " when the finger mapping was inverted at test. Examples of the pictures and the basic paradigm can be seen in Figure 1.

\section{Results}

Response consistency. In order to determine whether the subjects were switching responses appropriately, the consistency of their responses was measured by comparing the last response given to an item at study and the response indicated at test. If the response key mapping remained the same, the subjects should maintain the same response. In contrast, if the response mapping changed, in order to remain consistent, the subjects would have to change the keyed response. Overall, the group was highly consistent $(93 \%, S D=4.1 \%)$. However, a single subject 


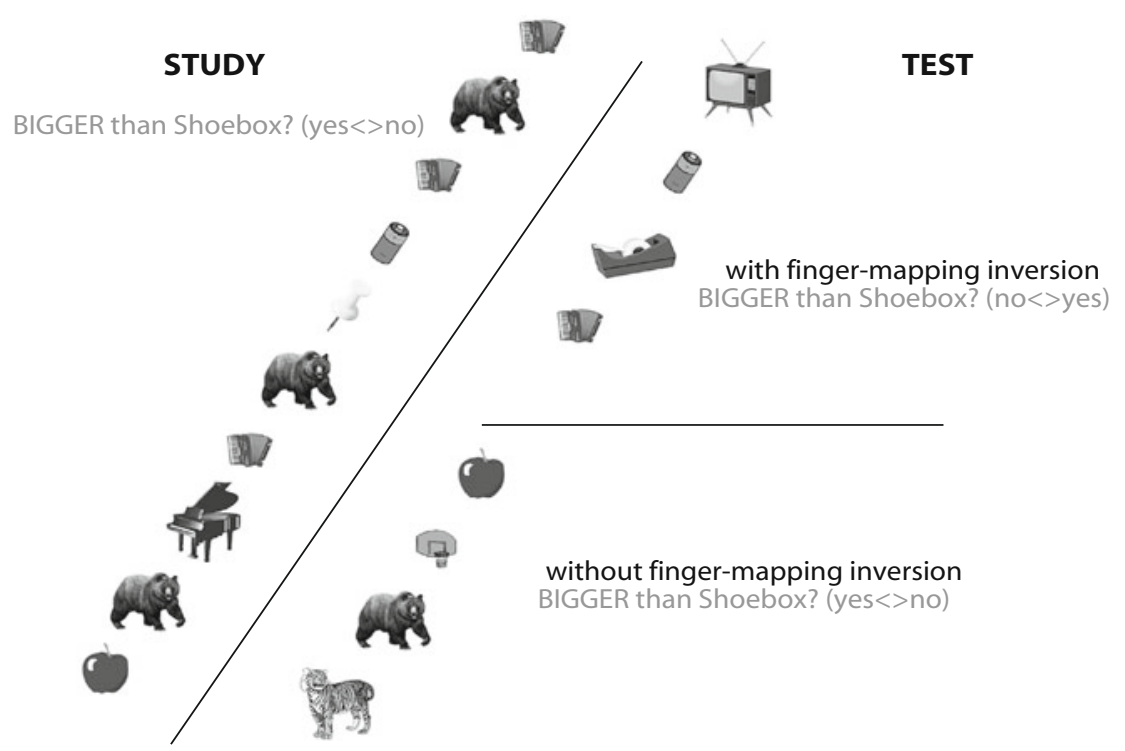

Figure 1. The general experimental paradigm. The subjects engaged in a study period in which they made classification judgments about items presented once or three times. At test, items presented once (low prime) and thrice (high prime) at study were presented along with novel items. Half of the time, at study, the subjects responded with the same finger mapping as that used during study, and half of the time, with an inverted mapping.

was more than two standard deviations below the mean $(77 \%)$, and that person's data was eliminated from the analysis. Because the origin of inconsistent responses is unclear, these responses were also eliminated ( $6 \%$ of items overall).

Study phase priming. Response times (RTs) during the study phase demonstrated facilitation across repetitions. An ANOVA examining RTs, with presentation (once, twice, or thrice) as the within-subjects variable, revealed a significant main effect $[F(2,28)=202.13, p<$ $.001]$, and pairwise $t$ tests indicated that all the conditions were significantly different from one another (mean RT for the first presentation $=1,127 \mathrm{msec}$, second presentation $=947 \mathrm{msec}$, and third presentation $=884 \mathrm{msec}$; all $p \mathrm{~s}<.001)$.

Finger mapping inversion. RTs across all the test block conditions can be seen in Table 1 . The effect of inverting the finger mapping on priming was examined in

Table 1

Mean Response Times (in Milliseconds) and Standard Errors for Experiment 1

\begin{tabular}{|c|c|c|c|c|}
\hline \multirow[b]{3}{*}{ Priming Condition } & \multicolumn{4}{|c|}{ Finger Mapping } \\
\hline & \multicolumn{2}{|c|}{ Same } & \multicolumn{2}{|c|}{ Inverted } \\
\hline & $M$ & $S E$ & $M$ & $S E$ \\
\hline \multicolumn{5}{|l|}{ Block 1} \\
\hline Novel & 1,078 & 56 & 1,054 & 56 \\
\hline Low prime & 966 & 65 & 960 & 59 \\
\hline High prime & 865 & 52 & 873 & 56 \\
\hline \multicolumn{5}{|l|}{ Block 2} \\
\hline Novel & 1,055 & 64 & 1,145 & 61 \\
\hline Low prime & 928 & 59 & 1,035 & 59 \\
\hline High prime & 890 & 50 & 963 & 60 \\
\hline
\end{tabular}

Test Block 1, where the contrast between same and inverted mapping is not confounded by number of repetitions or temporal order. Proportional priming scores were calculated [(novel - repeat)/novel] and submitted to a 2 $\times 2$ ANOVA with finger mapping (same or inverted) and priming condition (low prime or high prime) as withinsubjects factors. There was a main effect of priming condition $[F(1,14)=39.31, p<.001]$, which reflected the fact that priming in the high-prime condition was greater than that in the low-prime condition. Neither the main effect of finger mapping $[F(1,14)=1.80, p>.20]$ nor the finger mapping $\times$ priming condition interaction $[F(1,14)<1]$ was significant, indicating that finger-mapping inversion had no effect on the level of priming for either high or low primes. Follow-up $t$ testing revealed that priming in all the conditions was significantly greater than zero (all $p \mathrm{~s}<$ .001 ; see Figure 2, Block 1 same mapping and Block 1 inverted mapping).

In order to determine whether finger-mapping inversion disrupted response learning after a longer period with the original mapping, we compared the priming scores when the finger-mapping reversal occurred during the first test block with those when it was delayed until the second test block. A $2 \times 2$ repeated measures ANOVA with block ( 1 or 2 ) and priming condition (low prime or high prime) as factors again resulted only in a main effect of priming condition $[F(1,14)=35.60, p<.001]$, continuing to reflect the overall difference in priming scores for highand low-primed items. Again, there was no evidence of an effect of finger-mapping inversion, as evidenced by the fact that neither the main effect of mapping $[F(1,14)<1]$ nor any interaction involving mapping $[F(1,14)<1]$ was significant. This result indicated that there was no effect 


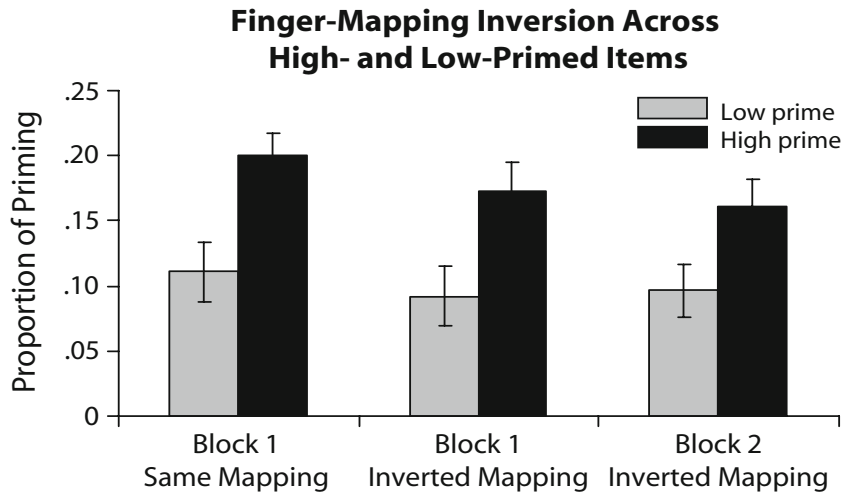

Figure 2. Proportional priming scores for low- and highprimed conditions across decision cue inversion in Test Blocks 1 and 2. Graphs represent proportions of priming, and error bars represent the standard error of the mean.

of finger-mapping inversion even after a longer period of responding with the original finger-mapping assignment (see Figure 2, Block 1 inverted mapping and Block 2 inverted mapping). ${ }^{1}$

\section{Discussion}

The results of Experiment 1 indicate that a change in the motor mapping of the response keys had no discernible effect on priming, either following a single presentation or following multiple presentations. Moreover, there was no effect of finger-mapping inversion, regardless of whether the change in decision cue occurred in Test Block 1 or in Test Block 2. Thus, having eliminated the confounds that were present in the previous study (Dobbins et al., 2004), our results are now in agreement with those obtained in other labs (Dennis \& Schmidt, 2003; Logan, 1990) and add to the evidence that the critical associative link that is forged during response learning is between a stimulus and its associated decision, rather than its motor response (Logan, 1990).

Having clarified the output level that is bound in response learning, we now will turn to an examination of the input level that is bound to the learned classification. It is possible that this binding occurs at the level of relatively abstract visual or even semantic representations; alternatively, the binding may be specific to the particular visual form that is presented during the study phase. One way to address this question is to evaluate response learning when different visual exemplars are presented at study and at test. Previous studies have shown that presenting different visual instantiations of the same object across repetitions reduces the level of priming, relative to when the same exemplar is repeated (Biederman \& Gerhardstein, 1993; Roediger \& Srinivas, 1993), and functional imaging studies have demonstrated that similar visual form manipulations reduce both behavioral- and "neuralpriming" effects in early visual-processing areas (Koutstaal et al., 2001; Simons, Koutstaal, Prince, Wagner, \& Schacter, 2003). However, it is important to note that in these studies, facilitation was not completely eliminated by substituting exemplars across repetitions. That is, although priming was significantly reduced, the subjects still responded significantly more quickly to these alternate exemplars than they did to completely new items. Whether this residual priming represents response learning is unknown.

If response learning reflects an association between a relatively abstract visual or semantic representation and a classification response, it should be preserved across visual form changes in repeated objects. Alternatively, if response learning reflects an association between a specific visual form and a classification response, response learning should be disrupted by changing the visual exemplar across repetitions. In Experiments 2A and 2B, we examined the effect of cue inversion on same- and differentexemplar repetitions in order to distinguish between these two possibilities.

\section{EXPERIMENT 2A}

\section{Method}

Subjects. Seventeen young native speakers of English (4 of them male, 13 female), with normal or corrected-to-normal vision, took part in the experiment. None of these individuals had participated in Experiment 1 . The subjects (mean age $=20$ years; range, $18-23$ ) were recruited through flyers and advertisements at local colleges and universities and received $\$ 10$ for their participation. The subjects were screened as described in Experiment 1. Written informed consent was obtained from each subject prior to the experimental session. The Human Subjects Committees of Boston University School of Medicine and the Veterans Affairs Healthcare System approved all the procedures.

Stimuli and Procedure. Three hundred items were selected for the present experiment from the items used in Experiment 1. Each of the selected items had a matching exemplar that differed visually but elicited the same name. The level of name correspondence between exemplars had previously been established behaviorally (Koutstaal et al., 2001). The items were divided into four groups, and item counterbalancing was accomplished by rotating the 300 items through the four conditions of once repeated, thrice repeated same exemplar, thrice repeated different exemplar, and novel. The objects were presented centrally, and viewing was approximately $75 \mathrm{~cm}$ from the screen. The experiment was conducted on a PC notebook computer running DMDX (software developed at Monash University and the University of Arizona by K. I. Forster and J. C. Forster).

The procedure was modified from Experiment 1 in two important ways. First, since previous studies had indicated that the most robust level of response learning occurs with three repetitions (Dobbins et al., 2004; Schnyer et al., 2006), the present experiment carried over into the test phase only items presented three times during study. Single items were included during the study phase so that the study conditions corresponded to those used in our previous experiments (Experiment 1 and Schnyer et al., 2006) and to ensure that not only repeated items were appearing by the end of the list. In addition, in order to simplify the analysis, each test phase was restricted to a single block with either cue inversion or same-cue conditions. The subjects engaged in six study-test cycles, with the test phases alternating between cue inversion and same cue. The order in which the two cue conditions were presented was counterbalanced across subjects.

During each study phase, the subjects made size judgments to 80 items total, consisting of 20 items repeated three times and 20 items presented once. The items were presented in pseudorandom order, with the items presented once evenly distributed throughout the study phase. None of the items presented once during study were seen during the test phase. The subjects were asked to make a size 
judgment by deciding whether the real-life object depicted in the picture was "bigger than a shoe box" and to indicate their decision by pushing a yes or a no key with the index or the middle finger, respectively, of their right hand.

Each test phase consisted of 30 randomly ordered pictures. Ten of these were exact copies of objects presented three times during study (same exemplar), 10 were visually different exemplars of objects presented three times during study (different exemplar), and 10 were objects not seen during study (novel). The division between the study and the test phases was indicated by a screen that instructed the subjects whether to continue with the same size decision or whether to invert that decision ("smaller than a shoe box").

The pictures were presented at a rate of one per $2 \mathrm{sec}$ and were accompanied at the bottom of the screen by the appropriate decision cue. Instructions appearing with the stimuli were "Bigger than a shoebox, $<$ yes/no $>$ " during the study phase and the same-cue test condition and "Smaller than a shoebox, $<$ no/yes $>$ " during the inverted-cue test condition.

\section{Results}

Response consistency. As in Experiment 1, the subjects were highly consistent in their responses $(94 \%, S D=$ $2.9 \%$ ), as evidenced by the nearly identical classification of items during the third study phase presentation and the test phase presentation. A single subject performed more than two standard deviations below the group mean $(86 \%)$, and that person's data were eliminated from the analysis. For all analyses of RTs, inconsistent responses were eliminated ( $5 \%$ of the items overall).

Study phase priming. As in our previous experiments, increasing repetition resulted in increased RT facilitation. An ANOVA examining RTs, with presentation (once, twice, or thrice) as the within-subjects variable, revealed a significant main effect $[F(2,30)=176.55, p<$ $.001]$, and post hoc testing indicated that all the conditions were significantly different from one another (mean RT for the first presentation $=949 \mathrm{msec}$, second presentation $=799 \mathrm{msec}$, and third presentation $=739 \mathrm{msec}$; all $p \mathrm{~s}<.001)$.

Decision cue inversion. The proportional priming scores across cue orientations and exemplar status are shown in Figure 3, and the RTs for all the conditions can be seen in Table 2. Priming in all the conditions was significantly greater than zero (all $p \mathrm{~s}<.005$ ). Proportional

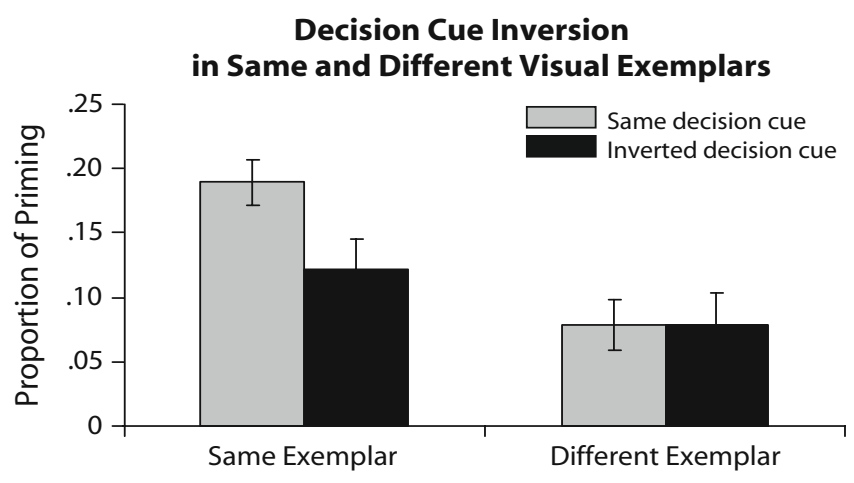

Figure 3. Proportional priming scores for same and different visual exemplars across both decision cues. Error bars represent the standard error of the mean.
Table 2

Mean Response Times (in Milliseconds) and Standard Errors for Experiment 2A

\begin{tabular}{|c|c|c|c|c|}
\hline \multirow[b]{3}{*}{ Priming Condition } & \multicolumn{4}{|c|}{ Decision Cue } \\
\hline & \multicolumn{2}{|c|}{ Same } & \multicolumn{2}{|c|}{ Inverted } \\
\hline & $M$ & $S E$ & $M$ & $S E$ \\
\hline Novel & 901 & 31 & 969 & 37 \\
\hline Same exemplar primed & 730 & 27 & 854 & 33 \\
\hline Different exemplar primed & 831 & 30 & 888 & 28 \\
\hline
\end{tabular}

priming scores were examined in a $2 \times 2$ repeated measures ANOVA with decision cue (same or inverted) and exemplar condition (same exemplar repeated or different exemplar repeated) as within-subjects factors. There was no overall effect of decision cue $[F(1,15)=2.04, p>$ $.15]$, whereas there was an effect of exemplar condition $[F(1,15)=38.30, p<.001]$, which reflected the fact that priming was greater for repetition of the same visual exemplar than for repetition of different visual exemplars. Most important, there was a significant decision cue $\times$ exemplar condition interaction $[F(1,15)=5.03, p<.05]$. For the same-exemplar repetitions, priming was greater in the same-cue than in the inverted-cue condition $[t(15)=$ $2.46, p<.05]$. By contrast, for different-exemplar repetitions, there was no difference in priming in the same-cue and inverted-cue conditions $[t(15)<1]$. Thus, there was no evidence that the portion of priming sensitive to decision cue inversion (and indicative of response learning) transfers across different visual exemplars of a studied item.

\section{EXPERIMENT 2B}

To determine whether the transfer of response learning across exemplars depends on the level of visual similarity, a separate group of subjects made visual similarity judgments for the exemplar pairs used in Experiment 2A. These judgments were then used to sort the items on the basis of visual similarity in order to determine whether sensitivity to cue inversion is dependent on the visual similarity of the exemplars.

\section{Method}

Subjects. Twelve young adults (10 of them female, 2 male), average age 23.6 years (range, 18-32), participated in the similarity ratings. None of these individuals had participated in Experiment 2A. The subjects were recruited through flyers and advertisements at local colleges and universities and received $\$ 10$ for their participation. The subjects were screened as described in Experiments 1 and 2A. Written informed consent was obtained from each subject prior to the experimental session. The Human Subjects Committees of Boston University School of Medicine and the Veterans Affairs Healthcare System approved all the procedures.

Stimuli and Procedure. Each of the 12 subjects saw all 300 image pairs that were utilized in Experiment 2A across three separate runs of 100 pairs each. Pairs of images were shown side by side at the center of the screen, and the subjects had a total of $20 \mathrm{sec}$ in which to rate how visually similar they thought the images to be. Once the rating was given for a particular item, the next pair was presented. Before beginning, the subjects were shown a few example pairs and were given these instructions: 
In this task you will see two images side by side and will be asked to rate how visually similar they are. There is no right or wrong answer. These images were used in a previous study and we just want to know how visually similar people think they are. You can base your similarity judgments on the shape, orientation and design of the images. However, please do not use the color or size of the images to make your similarity judgments. You will make your rating on a scale from 1 to 6 using this scale: 1-extremely dissimilar, 2-dissimilar, 3-slightly dissimilar, 4-slightly similar, 5-similar, 6-extremely similar.

\section{Results}

Mean similarity ratings were generated for each of the 300 pairs of items. These mean ratings were then used to sort the different exemplar conditions from Experiment 2A into five bins: Bin 1, items rated 1-1.99 (2\%); Bin 2, 2-2.99 (24\%); Bin 3, 3-3.99 (41\%); Bin 4, 4-4.99 $(81 \%)$; Bin 5, 5-6 (6\%). Bin 1 was dropped from the analysis because there were too few items. The RTs from Experiment 2A, sorted by similarity rating for the sameand inverted-cue conditions, can be seen in Table 3. Mean proportional priming scores from Experiment $2 \mathrm{~A}$ were reanalyzed in a $2 \times 4$ repeated measures ANOVA with decision cue (same or inverted) and level of judged similarity (Bin 2, Bin 3, Bin 4, or Bin 5) as within-subjects factors. There was no effect of decision cue orientation $[F(1,11)<1]$, but there was a significant effect of similarity rating $[F(3,33)=3.57, p<.01]$. Planned post hoc comparisons indicated that only the low similarity rating Bin 2 revealed less priming, relative to all the other bins $(p<.05)$, and priming was not significant for that bin $[t(15)<1$; see Figure 4]. Importantly, there was no evidence that the similarity rating interacted with the decision cue orientation $[F(3,33)<1]$. Thus, priming was unaffected by inversion of the decision cue, regardless of the visual similarity of the exemplars, suggesting that response learning did not transfer across any exemplars. A graph of the level of response facilitation across the level of visual similarity for both same and inverted response cues, along with examples of the pictures and their corresponding exemplars for Bin 2 and Bin 5 can be seen in Figure 4.

\section{Discussion}

Experiment 2B demonstrated that response learning in a classification task did not transfer across different visual exemplars of the same object, even when the perceptual changes were highly subtle. This is evident from

Table 3

Mean Response Times (in Milliseconds) and Standard Errors in Experiment $2 A$ Sorted Across the Four Similarity Rating Bins Generated in Experiment 2B

\begin{tabular}{cccccc}
\hline & \multicolumn{3}{c}{ Decision Cue } \\
\cline { 2 - 5 } Similarity Rating Bins & \multicolumn{2}{c}{ Same } & & \multicolumn{2}{c}{ Inverted } \\
\cline { 2 - 5 } \cline { 5 - 6 } & $M$ & $S E$ & & $M$ & $S E$ \\
\hline $5-6$ & 826 & 67 & 864 & 86 \\
$4-5$ & 821 & 34 & 879 & 44 \\
$3-4$ & 838 & 41 & 905 & 44 \\
$2-3$ & 911 & 38 & 962 & 30 \\
\hline
\end{tabular}

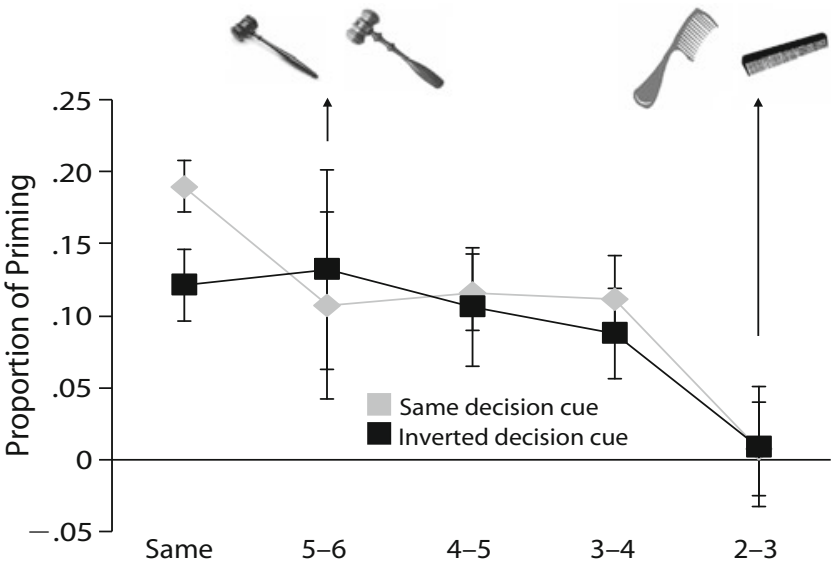

Figure 4. A graph of the proportional priming scores across levels of visual similarity between exemplars for both the same decision cue and the inverted decision cue. From left to right, values reflect repetitions of the same visual exemplar, followed by the Visual Similarity Rating Bins 5, 4, 3, and 2 for repetitions of different visual exemplars. Located above the graph are representative examples of the visual objects at the two extremes of the similarity ratings. Error bars represent the standard error of the mean.

the fact that decision cue inversion had no effect on the magnitude of priming for different exemplars. Furthermore, even when different exemplars were analyzed on the basis of subjective ratings of visual similarity, there was still no evidence of response learning, as was evident from a lack of any effects of decision cue inversion even for the exemplars judged to be most highly similar. Priming was observed across exemplars, however, and as in a previous study in which multiple study repetitions were used (Koutstaal et al., 2001), the magnitude of priming for different exemplars was significantly smaller than that exhibited for repeated same exemplars. The only exception to this was items judged to be very dissimilar; these items evidenced no priming at all. It is difficult to interpret why there was no priming across dissimilar exemplars, but one possibility is that these items had lower name agreement and, as such, a significant portion of them may have been considered fundamentally different items.

The present results provide the first evidence that response learning in an object classification task is perceptually highly specific and is not preserved across visually similar exemplars. It remains unknown whether there are other object-related perceptual parameters across which response learning might transfer. For instance, it is possible that response learning would not be disrupted by color changes (Cave, Bost, \& Cobb, 1996), changes in relative visual size (Biederman \& Gerhardstein, 1993), or depthrotation/mirror-imaging (Lawson, 2004). In the verbal domain, Logan et al. (1996) have examined the impact of color changes on classification learning. They found that classification learning for words was not disrupted by a change in the color in which the words were presented, unless the subjects were required to attend to the word color at study. Thus, the effect of featural changes on response 
learning may depend on the relevance of the featural change to the task. However, the correspondence between our work examining classification of visually presented objects and research with visually presented word stimuli (Logan et al., 1996) is not yet well established, and important differences may yet emerge.

Also relevant are findings of a study of instance learning that examined how repetition and visual similarity affect the speed of numerosity judgments to random dot patterns (Palmeri, 1997). In that study, subjects saw dot patterns repeatedly throughout a training phase and, in a transfer phase, were presented with dot patterns that varied in visual similarity to those in the training phase. As was expected, RTs decreased across the training phase, and response latencies during the transfer phase depended on the degree of visual similarity to the original patterns. The presence of instance learning was revealed by an examination of response latencies as a function of the number of dots in each pattern. Whereas early in learning latencies increased roughly linearly with the number of dots, at the end of training response latencies were independent of numerosity, suggesting that the subjects had learned a specific response to each pattern. For transfer patterns with moderate similarity, the slope of the function relating RT to numerosity fell in between that for new and repeatedly trained patterns. This finding might be taken as evidence that for some dot patterns, response learning is preserved despite some degree of visual change, whereas for other patterns, a counting algorithm needs to be applied anew. An alternative possibility is that for each pattern, performance reflects some combination of instance learning and application of the counting algorithm. For instance, a subset of dots within the altered array may retain the same visual pattern as that in the learning phase. This may allow a subject to rapidly retrieve a previous count for that cluster and then to continue counting the remaining dots. Importantly, on this view, instance learning will occur only for any cluster that does not undergo visual distortion. Regardless of the best account of Palmeri's findings, it is important to keep in mind that learning transfer was directly related to the degree of visual distortion, suggesting that instance learning is, indeed, disrupted by visual change.

The finding in the present study that response learning failed to transfer between different object exemplars, even when judged to have a high degree of visual similarity, can be understood in the context of a theoretical framework that postulates dissociable object representation subsystems for specific and abstract object codes. Previous work utilizing divided visual field presentations (Marsolek, 2004; Marsolek \& Burgund, 2003) has demonstrated that the right hemisphere shows greater priming for same than for different exemplars, whereas the left hemisphere shows equal priming for both. These findings have been interpreted as evidence for the existence of a specific object representation subsystem within the right hemisphere and an abstract object representation system within the left hemisphere (for related ideas, see Schacter, 1994). Further evidence for this notion comes from imaging studies, which have demonstrated that the right fusiform gyrus is differentially sensitive to repetition of specific exemplars (Koutstaal et al., 2001; Simons et al., 2003), whereas the left fusiform gyrus exhibits repetition-related changes more generally across exemplars. Within this framework, the findings of the present study suggest that the association between a response decision and object representation is established within a right-hemisphere-specific representation system. Future neuroimaging and/or divided field studies will be needed to confirm this neuroanatomical suggestion.

In the present study, all but the highly dissimilar exemplars showed priming, and the magnitude of priming was not directly tied to the nature of the decision cue. In addition, for same exemplars as well, there remained a level of priming that was not disrupted by decision cue inversion. These two findings point to a portion of the facilitation that operates on relatively abstract representations (Bowers, 2000), and this level of representation appears not to be linked directly to outcome decisions. The distinction between abstract representations, which mediate priming across exemplars, and specific representations, which mediate response learning, is adaptive, since each representational system allows for a different level of learning. In the case of the abstract system, generalization across exemplars enables establishment of a general organizational structure that allows for flexible application of novel classification and decision schemes. In contrast, when a specific item is repeatedly associated with a specific decision outcome, there are considerable computational savings associated with the rapid learning of these instances, and this is precisely what appears to be occurring in response learning.

Finally, although there is a well-formulated theoretical framework regarding abstract and specific representations and their influence on priming, we have reached the conclusions in our present work primarily through the presence of statistical independence between components. Because the empirical measure being used (RT) has undergone a number of transformations from the proposed underlying cognitive/neural components, any statistical differences could reflect variance due to these transformations, rather than independence of the components. Future converging evidence, neural dissociations, and wellformulated computational models will be needed in order to provide further support for the proposed framework.

\section{GENERAL DISCUSSION}

The present experiments suggest that multiple components contribute to priming and, in so doing, present a challenge for several existing theoretical frameworks that assume that a single mechanism accounts for all priming effects. A significant portion of the facilitation associated with the repetition of objects appears to involve the reliance on learned classification responses that bypass processes engaged during the initial classification. These learned responses do not reflect changes in object knowledge systems and, therefore, cannot easily be accommodated within abstractionist frameworks (Bowers, 2000) that argue for facilitation or tuning within object 
knowledge systems (Wiggs \& Martin, 1998). Instead, this component is consistent with an instance-learning account (Logan, 1990), which postulates that items come to be associated with a learned classification. In this regard, what we have previously referred to as response learning can perhaps be more accurately termed decision learning.

In addition to decision learning, there appears to be another component of repetition priming that operates across a certain level of visual dissimilarity, and this component is impervious to decision cue inversion. This component demonstrates features that are easily accommodated by an abstractionist framework (Bowers, 2000). By this framework, item repetition induces changes in abstract representations that are not tied to a specific encounter. This level of representation is general enough to allow facilitation to transfer across different visual presentations of the same item.

A question arises as to whether priming across different exemplars, which we take to reflect activation of abstract representations, might also be accommodated by an instance-learning framework (Tenpenny, 1995). For example, could it be that priming for different exemplars reflects binding of the decision to a lexical, rather than a visual, representation (Logan et al., 1996)? The majority of the visually presented items used in the present study are relatively easy to identify, and therefore, little attention is required to identify them. However, a small subset of items may be more difficult to identify, and therefore, additional attentional resources may be needed to name the object before the classification decision can be made (e.g., "is this item a nail or a broom stick?"). It could be argued that the additional effort associated with naming the item may result in a learned instance that reflects the binding between the name and the decision outcome. Such instances would transfer across exemplars and, thus, might provide a mechanism for different exemplar priming. Because bound instances at the lexical level would occur less frequently than bound instances at the visual level, the resulting facilitation would be less for different exemplars than for same exemplars.

Inconsistent with this account, however, is the fact that decision cue inversion had no effect on the level of facilitation for different exemplar items. If priming for these items reflected a name-decision linkage, one would expect an effect of cue inversion, because cue inversion disrupts the output level of the learned instance. Such disruption should occur regardless of whether the decision is bound to a specific visual representation or a lexical one. Thus, although it is possible to formulate different associations that could account for these results, it is more likely that priming for different exemplars, both prior to and after cue inversion, is not tied to specific instances. Priming for different exemplars, as well as the component of same-exemplar priming that is unaffected by cue inversion, is more easily accommodated in the context of an abstractionist framework. In this regard, our view diverges from one that postulates that repetition priming and the automaticity that emerges from multiple repetitions stem from a unitary mechanism (Logan, 1990).
An unresolved issue with regard to the two components of priming evident in the present experiments is whether they are independent or interactive. One possibility is that they reflect independent parallel pathways to decision output. In this case, the level of facilitation exhibited would reflect the component that dominates the "race" to decision output (Logan, 1988; Marsolek, 2004). The division of abstract and specific representations as subserved by parallel left- and right-hemisphere pathways is consistent with this view (Marsolek, 2004). Alternatively, these components could be additive, with the additional facilitation associated with multiple repetitions added to the abstract component associated with a single repetition. Future experiments will be required to resolve this issue.

The sensitivity of classification learning to the correspondence between study and test formats indicates a high degree of specificity in the establishment and utilization of learned responses. Such hyperspecificity (Schacter, 1985 ) is consistent with the notion that response learning is episodic in nature. In fact, the working assumption is that instance learning reflects storage and retrieval of episodic representations (see Tenpenny, 1995, for a discussion of this issue). With results similar to those in Experiments $2 \mathrm{~A}$ and $2 \mathrm{~B}$, a number of studies have demonstrated that episodic representations encode visually specific information and, thereby, are sensitive to changes in visual format (Biederman \& Cooper, 1991; Lawson, 2004; Srinivas \& Verfaellie, 2000). In addition, our own work has already demonstrated that response learning is impaired in MTL amnesics (Schnyer et al., 2006). Within the realm of repetition priming, however, the key question is whether response learning reflects conscious intentional retrieval of previous item-decision associations (Schacter, Bowers, \& Booker, 1989) or an unconscious unintentional form of memory that, nonetheless, is MTL-dependent and episodic in nature (Chun \& Phelps, 1999). In this regard, evidence is beginning to emerge suggesting that response learning reflects an unintentional form of memory. In a series of studies exploring the laterality dissociations between abstract and specific visual representations in word stem completion priming, Marsolek (2004) examined both case-specific priming and case-specific explicit memory. He obtained a clear dissociation between the characteristics of the implicit and the explicit tasks, indicating that visually specific priming cannot be attributed to explicit retrieval. In addition, a recent electrophysiological study of response learning in our lab (Schnyer, Dobbins, Nicholls, Schacter, \& Verfaellie, 2005) has demonstrated that prior response associations are retrieved approximately $230 \mathrm{msec}$ after stimulus presentation. Event-related potential research has revealed a reliable parietally located old-new effect that indexes recollection and occurs between 400 and $800 \mathrm{msec}$ post-stimulus-onset (Gonsalves \& Paller, 2000; see Rugg, 1995, for a review). Therefore, the time course of response association retrieval appears considerably earlier than effects associated with conscious retrieval of episodic memories. Finally, intuition tells us that less effort would be required to perform the relatively simple object size judgment than would be involved in 
explicitly retrieving a prior classification (Jacoby, 1991) and then utilizing that information to guide responses. Final resolution of this question will require continuing experiments that seek clear explicit/implicit retrieval dissociations in response learning.

In addition to the decision specificity examined here, across the repetition priming literature, there are a number of other instances of hyperspecificity in priming, including stimulus specificity and associative specificity (Schacter et al., 2004). Whether these other forms of specificity similarly reflect a form of decision learning remains to be determined, as does their dependence on the MTL (Marsolek, 2004). In this regard, it is useful to consider several characteristics of decision learning. First and most apparent is the inflexibility of the stimulus and decision components that become associated. Alteration of either of these components challenges the established association, thus disrupting learning. Second, in contrast to priming of abstract representations, decision learning is enhanced with multiple repetitions, although such learning may reach a maximal level of facilitation relatively quickly (Buckner et al., 1998; Logan, 1990). Finally, decision learning appears to depend on associative-learning mechanisms mediated by the MTLs, as demonstrated by the fact that instance learning is impaired in patients with amnesia (Schnyer et al., 2006). Future studies can utilize these basic characteristics in order to determine the extent to which a similar learning mechanism is responsible for the specificity effects evident across a wide range of repetition-priming paradigms.

\section{AUTHOR NOTE}

This work was supported by Grants K23MH64004 (D.M.S.) and P50 NS26985 and MH57681 (M.V.) to Boston University, by the Medical Research Service of the Department of Veterans Affairs and by AG08441 (D.L.S.) to Harvard University. We thank two anonymous reviewers and Chad Marsolek for their helpful comments on this work. Correspondence concerning this article should be addressed to D. M. Schnyer, Department of Psychology, A8000, University of Texas, Austin, TX 78712 (e-mail: schnyer@psy.utexas.edu).

\section{REFERENCES}

Biederman, I., \& Cooper, E. E. (1991). Evidence for complete translational and reflectional invariance in visual object priming. Perception, 20, 585-593.

Biederman, I., \& Gerhardstein, P. C. (1993). Recognizing depthrotated objects: Evidence and conditions for three-dimensional viewpoint invariance. Journal of Experimental Psychology: Human Perception \& Performance, 19, 1162-1182.

BowERs, J. S. (2000). In defense of abstractionist theories of repetition priming and word identification. Psychonomic Bulletin \& Review, 7, 83-99.

Buckner, R. L., Goodman, J., Burock, M., Rotte, M., Koutstaal, W., SCHACTER, D., ET AL. (1998). Functional-anatomic correlates of object priming in humans revealed by rapid presentation event-related fMRI. Neuron, 20, 285-296.

Burgund, E. D., \& Marsolek, C. (1997). Letter-case-specific priming in the right cerebral hemisphere with a form-specific perceptual identification task. Brain \& Cognition, 35, 239-258.

Cave, C. B., Bost, P. R., \& CobB, R. E. (1996). Effects of color and pattern on implicit and explicit picture memory. Journal of Experimental Psychology: Learning, Memory, \& Cognition, 22, 639-653.

Chun, M. M., \& Phelps, E. A. (1999). Memory deficits for implicit contextual information in amnesic subjects with hippocampal damage. Nature Neuroscience, 2, 844-847.
Dennis, I., \& Schmidt, K. (2003). Associative processes in repetition priming. Journal of Experimental Psychology: Learning, Memory, \& Cognition, 29, 523-538.

Dobbins, I. G., Schnyer, D. M., Verfaellie, M., \& Schacter, D. L. (2004). Cortical activity reductions during repetition priming can result from rapid response learning. Nature, 428, 316-319.

FISK, A. D., \& SCHNEIDER, W. (1984). Consistent attending versus consistent responding in visual search: Task versus component consistency in automatic processing development. Bulletin of the Psychonomic Society, 22, 330-332.

Gonsalves, B., \& Paller, K. A. (2000). Brain potentials associated with recollective processing of spoken words. Memory \& Cognition, 28, 321-330

JACOBY, L. L. (1991). A process dissociation framework: Separating automatic from intentional uses of memory. Journal of Memory \& Language, 30, 513-541.

Koutstaal, W., Wagner, A. D., Rotte, M., Maril, A., Buckner, R. L., \& Schacter, D. L. (2001). Perceptual specificity in visual object priming: Functional magnetic resonance imaging evidence for a laterality difference in fusiform gyrus. Neuropsychologia, 39, 184199.

LAWSON, R. (2004). Depth rotation and mirror-image reflection reduce affective preference as well as recognition memory for pictures of novel objects. Memory \& Cognition, 32, 1170-1181.

Logan, G. D. (1988). Toward an instance theory of automatization. Psychological Review, 95, 492-527.

LogAN, G. D. (1990). Repetition priming and automaticity: Common underlying mechanisms? Cognitive Psychology, 22, 1-35.

Logan, G. D., \& Etherton, J. L. (1994). What is learned during automatization? The role of attention in constructing an instance. Journal of Experimental Psychology: Learning, Memory, \& Cognition, 20, 1022-1050.

Logan, G. D., Taylor, S. E., \& Etherton, J. L. (1996). Attention in the acquisition and expression of automaticity. Journal of Experimental Psychology: Learning, Memory, \& Cognition, 22, 620-638.

MARSOLEK, C. [J.] (2004). Abstractionist versus exemplar-based theories of visual word priming: A subsystems resolution. Quarterly Journal of Experimental Psychology, 57A, 1233-1259.

Marsolek, C. J., \& Burgund, E. D. (2003). Visual recognition and priming of incomplete objects: The influence of stimulus and task demands. In J. S. Bowers \& C. J. Marsolek (Eds.), Rethinking implicit memory (pp. 139-156). Oxford: Oxford University Press.

PALMERI, T. J. (1997). Exemplar similarity and the development of automaticity. Journal of Experimental Psychology: Learning, Memory, \& Cognition, 23, 324-354.

Roediger, H. L., III, \& SRINIVAs, K. (1993). Specificity of operations in perceptual priming. In P. Graf \& M. E. Masson (Eds.), Implicit memory: New directions in cognition, development and neurspsychology (pp. 17-48). Hillsdale, NJ: Erlbaum.

RUGG, M. D. (1995). Event-related potential studies of human memory. In M. S. Gazzaniga (Ed.), The cognitive neurosciences (pp. 789-801). Cambridge, MA: MIT Press.

Schacter, D. L. (1985). Multiple forms of memory in humans and animals. In N. M. Weinberger, J. L. McGaugh, \& G. Lynch (Eds.), Memory systems of the brain (pp. 351-379). New York: Guilford.

SCHACTER, D. L. (1994). Priming and multiple memory systems: Perceptual mechanisms of implicit memory. In D. L. Schacter \& E. Tulving (Eds.), Memory Systems 1994 (pp. 233-268). Cambridge, MA: MIT Press.

Schacter, D. L., Bowers, J., \& Booker, J. (1989). Intention, awareness, and implicit memory: The retrieval intentionality criterion. In S. Lewandowsky, J. C. Dunn, \& K. Kirsner (Eds.), Implicit memory: Theoretical issues (pp. 47-65). Hillsdale, NJ: Erlbaum.

Schacter, D. L., Dobbins, I. G., \& Schnyer, D. M. (2004). Specificity of priming: A cognitive neuroscience perspective. Nature Reviews Neuroscience, 5, 853-862.

Schnyer, D. M., Dobbins, I. G., Nicholls, L., Schacter, D. L., \& Verfaellie, M. (2005, November). Rapid decision learning alters repetition-related components of left frontal and temporal regions: Evidence from meg recordings during repetition priming. Paper presented at the 35th Annual Meeting of the Society for Neuroscience, Washington, DC

Schnyer, D. M., Dobbins, I. G., Nicholls, L., Schacter, D. L., \& 
Verfaellie, M. (2006). Rapid response learning in amnesia: Delineating associative learning components in repetition priming. Neuropsychologia, 44, 140-149.

Simons, J. S., Koutstaal, W., Prince, S., Wagner, A. D., \& Schacter, D. L. (2003). Neural mechanisms of visual object priming: Evidence for perceptual and semantic distinctions in fusiform cortex. NeuroImage, 19, 613-626.

SRINIVAS, K., \& Verfaellie, M. (2000). Orientation effects in amnesics' recognition memory: Familiarity-based access to object attributes. Journal of Memory \& Language, 43, 274-290.

TenPenny, P. L. (1995). Abstractionist versus episodic theories of repetition priming and word indentification. Psychonomic Bulletin \& Review, 2, 339-363.

Vaidya, C. J., Gabrieli, J. D. E., Verfaellie, M., Fleischman, D., \& AsKARI, N. (1998). Font-specific priming following global amnesia and occipital lobe damage. Neuropsychology, 12, 183-192.
Wiggs, C. L., \& Martin, A. (1998). Properties and mechanisms of perceptual priming. Current Opinions in Neurobiology, 8, 227-233.

\section{NOTE}

1. The same mapping conditions in Test Block 2 were not analyzed, since this condition was confounded by being preceded by a test block where the finger mapping had been inverted. Priming continued to be significant in this block, and there continued to be a difference between low- and high-primed items (mean low prime $=.12, S D=.06$; mean high prime $=.16, S D=.08$ ).

(Manuscript received November 11, 2005; revision accepted for publication May 11, 2006.) 